\title{
Deep vein thrombosis in arthroscopic surgery and chemoprophylaxis recommendation in an Asian population
}

Kuei Siong Andy $\underline{Y e o}^{1}$, MBBS, FRCS, Wen Siang Kevin $\underline{\operatorname{Lim}}^{1}$, MBBS, Yee Han Dave $\underline{\operatorname{Lee}}^{1}$, MBBS, FRCS

INTRODUCTION There are currently no guidelines supporting the use of routine chemoprophylaxis to prevent deep vein thrombosis (DVT) in arthroscopic surgery. Studies and meta-analysis show opposing views on its routine use in arthroscopy. This study aimed to examine the incidence of DVT in a prospective cohort of knee arthroscopy and knee arthroplasty patients, and to analyse the risk factors contributing to DVT.

METHODS All patients scheduled to undergo knee arthroscopy or arthroplasty over a two-year period were included. A standardised regimen of postoperative mechanical prophylaxis and rehabilitation was applied to all patients. Only patients who were postoperatively symptomatic were referred for ultrasonography. DVT incidence was calculated, and univariate and multivariate analyses of the risk factors were performed.

RESULTS The overall incidence of DVT was $0.5 \%$ among the 1,410 arthroscopy patients and $3.1 \%$ among the 802 arthroplasty patients. The incidence of proximal DVT among the arthroscopy and arthroplasty patients was $0.4 \%$ and $1.1 \%$, respectively. Multivariate analysis showed that age was the only significant predictor of DVT incidence. Using the receiver operating characteristic method, the cut-off age for the arthroscopy and arthroplasty patients was 52 years, while that for the arthroscopy patients only was 40 years (increased risk of DVT: 5.46 and 6.44 times, respectively; negative predictive value: $99.7 \%$ and $99.8 \%$, respectively).

CONCLUSION DVT incidence among Asian arthroplasty and arthroscopy patients remains low, even without chemoprophylaxis. Since age was found to be a significant risk factor for DVT, DVT prophylaxis can be considered for patients in high-risk age groups.

Keywords: arthroplasty, arthroscopy, Asian, chemoprophylaxis, DVT

\section{INTRODUCTION}

Deep vein thrombosis (DVT), a known complication of lower limb surgery, and its sequelae of pulmonary embolism (PE) can be prevented via mechanical or chemical thromboprophylaxis. Guidelines by the American College of Chest Physicians (ACCP) and the American Academy of Orthopaedic Surgeons (AAOS) $)^{(1)}$ are used to determine the prophylaxis regimen for patients undergoing lower limb surgery. However, thromboprophylaxis is still avoided, at times, due to the possible side effects of anticoagulation. Furthermore, there are no recommendations on DVT prophylaxis after knee arthroscopic surgery.

The incidence of DVT after knee arthroscopy is not as wellstudied as the incidence of DVT after knee arthroplasty. According to previous studies, the incidence of DVT among patients who underwent knee arthroscopy ranged from 4.1\%-17.9\%; in these studies, all patients underwent routine ultrasonography or venography for diagnosis. ${ }^{(2-5)}$ The variance in the incidence rates could have been due to the type of surgery performed, as the aforementioned studies examined specific subgroups of arthroscopic surgeries. For instance, the study by Wirth et al, (2) which reported a low incidence (i.e. $4.1 \%$ ) of DVT, involved arthroscopic patients who underwent shorter surgeries (i.e. partial meniscectomies, removal of foreign bodies and resurfacing of cartilage). On the other hand, the study by Dong et $\mathrm{al}^{\left({ }^{(5)}\right.}$ which reported a DVT incidence of $12.1 \%$, involved patients who underwent anterior cruciate ligament reconstruction.

In studies where proximal and distal DVT were differentiated, the incidence of proximal DVT was $1.45 \%-2.1 \%$ and the overall DVT rates were reported to be $6.8 \%-9.9 \%$. ${ }^{(6,7)}$ To the best of our knowledge, there are neither systematic reviews nor meta-analyses of studies involving DVT incidence for arthroscopic surgeries conducted in Asian populations. Thus, the purpose of the present study was to: (a) compare rates and relative risk of DVT and PE between two major types of surgery; (b) analyse DVT patterns in knee arthroscopy in the local population; and (c) identify trends and possible risk stratification that can inform the decision on whether patients undergoing knee arthroscopy should receive prophylaxis.

\section{METHODS}

This study was a prospective cohort study. Patients admitted to Changi General Hospital, Singapore, for knee surgery between January 2011 and December 2013 were eligible for inclusion in this study. Patients who underwent knee arthroplasty and arthroscopy were included; patients with septic arthritis and those who underwent trauma surgery and implant removal were excluded.

A total of 2,212 patients were enrolled. All patients followed a standardised rehabilitation protocol of mechanical prophylaxis

${ }^{1}$ Department of Orthopaedics, Changi General Hospital, Singapore

Correspondence: Dr Kuei Siong Andy Yeo, Associate Consultant, Department of Orthopaedics, Changi General Hospital, 2 Simei Street 3, Singapore 529889.

andy_yeo_ks@cgh.com.sg 
and early ambulation on the first postoperative day. They were monitored for clinical signs and symptoms of DVT during the postoperative period. Patients who had symptoms that were suspicious for DVT (e.g. lower limb pain or swelling) underwent Doppler ultrasonography to check for the presence of DVT. If the patient was found to be positive for DVT, its location (proximal or distal) was noted. Computed tomography (CT) was performed for patients who had clinical signs and symptoms that were suspicious for PE; any positive findings on $\mathrm{CT}$ were recorded. Statistical analyses were performed using IBM SPSS Statistics for Windows version 22.0 (IBM Corp, Armonk, NY, USA). Nonparametric tests, such as Mann-Whitney $U$ test and Spearman correlation, were used in the data analysis. A p-value $<0.05$ was considered statistically significant.

\section{RESULTS}

A total of 2,212 patients (1,390 men and 822 women) were included in this study. Their median age was 44.3 (interquartile range [IQR] 23-62) years. 802 patients underwent knee arthroplasty (i.e. knee arthroplasty group), while 1,410 underwent knee arthroscopy (i.e. knee arthroscopy group). The median age of patients in the knee arthroplasty group was 65 (IQR 60.00-72.00) years, while that of the knee arthroscopy group was 27 (IQR 21.00-41.25) years. The median ages of the two groups were significantly different (mean difference 33.10 years, confidence interval $[\mathrm{Cl}] 32.04-34.15$; $\mathrm{p}<0.01$ ), which was expected due to the nature of the surgery.

In the knee arthroplasty group, 25 (3.1\%) patients were found to be positive for DVT, compared to $7(0.5 \%)$ patients in the knee arthroscopy group ( $p<0.01$ using Pearson chi-square test) (Table I). Univariate logistic regression comparing the relative risk of DVT between these two groups showed that patients who underwent arthroplasty were 6.334 times more likely than patients who underwent arthroscopy to develop DVT as a postoperative complication $(p<0.01)$. However, this relationship was not found to be significant $(p=0.06)$ in the multivariate logistic regression, which included other factors such as ethnicity, gender and age.

In the knee arthroplasty and knee arthroscopy groups, 9 (1.1\%) patients and $6(0.4 \%)$ patients had proximal DVT, respectively. In contrast to the overall DVT rates, the difference in the proximal DVT rates was not found to be statistically significant $(p=0.06$, using Pearson chi-square test). Univariate analysis of the proximal DVT rates showed that the odds ratio of developing DVT in the knee arthroplasty group was 2.609. Although this relationship was near significance, it was not statistically significant $(p=0.07)$. The number of patients with PE was $2(0.2 \%)$ and $1(0.1 \%)$ in the arthroplasty and arthroscopy groups, respectively; this difference was not found to be statistically significant ( $p=0.30$, using Pearson chi-square test).

Based on the data obtained, a cut-off age was determined using the receiver operating characteristic (ROC) method. The patients from both groups were stratified into $>52$ years and $\leq 52$ years. The use of this cut-off age showed a statistically significant difference in DVT rates between the two groups, with the Pearson chi-square test result of $p<0.01$. The negative predictive value (NPV) was $99.7 \%$ and the positive predictive
Table I. Comparison of the rates of deep vein thrombosis (DVT), proximal DVT and pulmonary embolism (PE) between the patients who underwent knee arthroscopy and knee arthroplasty.

\begin{tabular}{lccc}
\hline Variable & \multicolumn{2}{c}{ No. (\%) } & p-value \\
\cline { 2 - 3 } & $\begin{array}{c}\text { Knee } \\
\text { arthroscopy } \\
(\mathbf{n}=\mathbf{1 , 4 1 0 )}\end{array}$ & $\begin{array}{c}\text { Knee } \\
\text { arthroplasty } \\
(\mathbf{n}=\mathbf{8 0 2})\end{array}$ & \\
\hline Gender & $1,162(82.4)$ & $228(28.4)$ & $<0.01$ \\
$\quad$ Male & $248(17.6)$ & $574(71.6)$ & \\
Female & $32.14 \pm 13.61$ & $65.68 \pm 9.14$ & $<0.01$ \\
Age* $(\mathbf{y r})$ & $7(0.5)$ & $25(3.1)$ & $<0.01$ \\
DVT & $6(0.4)$ & $9(1.1)$ & 0.06 \\
Proximal DVT & $1(0.1)$ & $2(0.2)$ & 0.30 \\
PE & & & \\
\hline
\end{tabular}

* Data presented as mean \pm standard deviation.

value (PPV) was $12.5 \%$ for overall DVT rates. Multivariate analysis showed a 5.5-fold (Cl 1.062-28.015) increase in risk of overall DVT in patients aged $>52$ years $(p=0.042)$. When the same cut-off age was applied to proximal DVT only, the result was significant ( $p=0.04$, using Pearson chi-square test); the NPV and PPV were $99.8 \%$ and $20.0 \%$, respectively. This result was similar when the same cut-off age was applied to the PE rates; $p=0.05$, using Pearson chi-square test, and the NPV and PPV were $100.0 \%$ and $0 \%$, respectively.

The ROC of the knee arthroscopy group alone showed an area value of 0.823 . Using the ROC method, the cut-off age for the stratification of this patient group was 40 years. In terms of overall DVT rates, multivariate analysis did not show any significance except for the stratified age of 40 years. The odds ratio was 6.44 (Cl 1.18-35.29) for patients aged $\geq 40$ years compared to those aged $<40$ years $(p=0.03)$. The NPV and PPV were $99.8 \%$ and $1.3 \%$, respectively, and $p<0.01$, using Pearson chi-square test. In terms of proximal DVT, patients who were aged $\geq 40$ years were found to have 12.58 times $(\mathrm{Cl} 1.39-113.67)$ the risk of developing proximal DVT as compared to those aged $<40$ years $(p=0.02$; Table II). The NPV and PPV were $99.9 \%$ and $1.3 \%$, respectively, and the p-value was 0.02 using Fisher's exact test.

\section{DISCUSSION}

The ACCP and AAOS guidelines for DVT prophylaxis are most widely accepted.(1) In the ACCP guidelines, antithrombotic prophylaxis lasting 10-14 days is recommended over no therapy (Grade 1C evidence) for total hip arthroplasty, total knee arthroplasty and hip fracture surgery. Antithrombotic prophylaxis is not recommended for patients undergoing arthroscopic surgery if they do not have a prior history of DVT (Grade 2B evidence). On the other hand, the AAOS guideline is not as specific and has a moderate recommendation for the use of pharmacologic and mechanical agents for prophylaxis in patients undergoing hip and knee arthroplasty surgery.

In the present study, our univariate analysis suggested that the DVT rate was significantly higher in the arthroplasty group than the arthroscopy group. However, when other factors were taken into account in the multivariate analysis, the observed difference became insignificant, suggesting that another stronger factor may 
Table II. Results of the logistic regression analysis conducted to analyse the factors for proximal deep vein thrombosis among the patients in the arthroscopy group.

\begin{tabular}{|c|c|c|c|c|}
\hline \multirow[t]{2}{*}{ Factor } & \multicolumn{2}{|c|}{ Unadjusted } & \multicolumn{2}{|c|}{ Adjusted } \\
\hline & B (95\% CI) & p-value & B (95\% CI) & p-value \\
\hline Gender & $2.35(0.43-12.92)$ & 0.33 & $1.11(0.19-6.36)$ & 0.91 \\
\hline \multicolumn{5}{|l|}{ Ethnicity } \\
\hline Chinese & - & - & - & - \\
\hline Malay & $1.08(0.18-6.49)$ & 0.93 & $1.16(0.19-7.00)$ & 0.87 \\
\hline Others & 0 & 0.99 & 0 & 0.99 \\
\hline Age $\geq 40 \mathrm{yr}$ & $12.55(1.40-112.81)$ & 0.02 & $12.58(1.39-113.67)$ & 0.02 \\
\hline
\end{tabular}

$\mathrm{Cl}$ : confidence interval

be responsible for the observed difference. As differences in the gender and ethnicity of the two groups were not significant, age was explored as a factor using the ROC method in the knee arthroscopy group. We found that patients aged $\geq 40$ years had a significantly higher relative risk of developing DVT, proximal DVT and PE.

The incidence of DVT in the arthroscopy group was $0.5 \%$ in the present study. This incidence is lower than those reported in studies by Wirth et al ${ }^{(2)}(4.1 \%$ incidence among 111 control patients) and Michot et $\mathrm{al}^{(3)}$ (15.6\% incidence among 64 control patients). In their studies, DVT was diagnosed on routine ultrasonography; however, in the study by Wirth et al, the incidence of clinical detection of DVT was $2.7 \%$, which is still higher than the incidence observed in the present study. In both of these studies, only distal DVT was detected. A study by Williams et $\mathrm{al}^{(8)}$ observed a DVT incidence of $3.5 \%$; their patients were diagnosed on routine ultrasonography and all of the patients with DVT were asymptomatic. In a meta-analysis by Ilahi et $\mathrm{al}_{,}{ }^{(7)}$ the pooled incidence was $9.9 \%$, but all cases were diagnosed on venography or ultrasonography. In contrast, Dahl et $\mathrm{al}^{(9)}$ studied almost 5,000 patients and the incidence of clinically symptomatic DVT (not diagnosed on routine scan) in the arthroscopic surgery arm was $0.6 \%$.

A systematic review of 56 articles by Graham et al ${ }^{(10)}$ showed a wide variance in the reported incidence of DVT in arthroscopic surgery. The authors attributed the variation to the large heterogeneity of the study populations, differences in the inclusion and exclusion criteria, and the different diagnostic methods used. The six large-scale retrospective studies that they analysed had incidences ranging from $0.12 \%$ to $0.8 \%$, which is similar to the rates of DVT in arthroscopic surgery observed in the present study. Like our study, these six studies only considered clinically symptomatic DVT.

Compared to knee arthroplasty, less evidence on DVT incidence and prophylaxis for knee arthroscopy has been reported. The meta-analysis conducted by Sun et $\mathrm{al}^{\left({ }^{(6)}\right.}$ on the effect of low-molecular-weight heparins (LMWH) on proximal DVT showed a $1.2 \%$ absolute risk reduction for patients on $\mathrm{LMWH}$ as compared to those who did not receive prophylaxis. The metaanalysis was mainly based on Level IV studies. However, as the overall rates were low $(1.5 \%$ of the 914 patients in the control population), the authors were unable to make any meaningful conclusion or recommendations on the routine use of LMWH for patients undergoing arthroscopic surgeries.

Although small-scale studies ${ }^{(2,3)}$ recommend the use of routine prophylaxis due to results that show a decrease in relative risk, this was not borne out by large analyses of adverse events. A 2008 meta-analysis by Ramos et $\mathrm{al}^{(11)}$ analysed four trials involving a total of 527 patients. Although the relative risk of thrombotic events was found to be 0.16 (Cl 0.05-0.52) in the intervention group, the authors also found a higher incidence of adverse events $(n=66)$ in this group; in fact, the number of adverse events exceeded the number needed to harm $(n=20)$ in the analysis. All thrombotic events observed were distal DVT, except for one PE. Thus, the authors concluded that although $\mathrm{LMWH}$ reduced the incidence of DVT, there is no strong evidence that thromboprophylaxis is effective and safe for patients undergoing knee arthroscopic surgery.

Aside from evidence-based decision-making, a survey on clinical practices revealed that the practice of administering routine chemoprophylaxis in arthroscopic surgeries varied between countries $(17 \%-96 \%$ of the respondents indicated that they would perform routine chemoprophylaxis in arthroscopic surgeries). ${ }^{(10)}$ Thus, there is a need to further stratify patients who are undergoing knee arthroscopic surgery so that better decisions can be made on whether chemical thromboprophylaxis should be used.

The results of the multivariate analysis of the present study showed that there were no risk factors associated with DVT incidence other than the cut-off age of 40 years. Using this cutoff age, the NPVs of those developing DVT (overall or proximal only) were high (99.7\%-99.8\%) and significant. Clinically, this translates to the fact that patients aged $<40$ years have a low risk of developing DVT and that there is no need to consider chemoprophylaxis in this group of patients.

The DVT incidence of the patients undergoing knee arthroplasty was $3.1 \%$ in the present study. This is comparable to larger-scale studies that reported clinically symptomatic DVT incidences of $2.1 \%, 2.8 \%$ and $3.7 \%$ for patients who underwent arthroplasty. ${ }^{(9,12,13)}$ However, the patients in those studies had both mechanical and chemical thromboprophylaxis, while the patients in the present study only had mechanical prophylaxis.

We found that the rates observed in our study are comparable to other studies that involved Asian populations and in which routine chemoprophylaxis was not performed. In a study by Bin Abd Razak et al, ${ }^{(14)}$ the authors reported that the rate of clinically 
symptomatic DVT of patients who underwent knee arthroplasty was $0.5 \%$. Low et $\mathrm{al}^{(15)}$ reported a $2 \%$ incidence of clinically symptomatic DVT, while a meta-analysis by Lee et $\mathrm{al}^{\left({ }^{(16)}\right.}$ showed a $1.9 \%$ incidence of symptomatic DVT. Lee et al ${ }^{(16)}$ showed that the incidence of DVT would be as high as $40.4 \%$ if asymptomatic DVT was included. Thus, we concluded that as our findings are comparable, this study reflects the actual situation for Asian patients undergoing arthroplasty. As the incidence of DVT is low even without chemoprophylaxis, it may only be needed for patients with significant DVT risk factors and who are of an advanced age, which was identified as a statistically significant factor in the present study.

The strength of the present study lies in the large numbers of patients in both the knee arthroplasty and knee arthroscopy groups. As the present study was conducted at a single institution, confounders such as surgical procedures and postsurgical management as well as mechanical prophylaxis and postoperative rehabilitation were similarly applied, giving some measure of control for the other factors. Also, our findings reflect the local context and the incidence of symptomatic DVT in Singapore; this incidence can vary greatly among populations from different countries.

One limitation of the present study was the diagnosis of only symptomatic DVT, which may have been lower than the incidence of actual DVT; however, DVT studies generally report either asymptomatic or symptomatic DVT. We found that our results, in terms of DVT incidence, were comparable to those of other studies that also examined symptomatic DVT. We avoided comparing the DVT incidence of the present study with those of studies with asymptomatic DVT. In general, studies with asymptomatic DVT also had smaller sample sizes than studies with symptomatic DVT. A further limitation of the present study was that the data of patients who presented to another hospital for DVT may not have been included. However, as our data collection method included two other regional hospitals, it would have mitigated this limitation. Furthermore, the focus of the present study was on immediate or early postoperative symptomatic DVT, which would have been noted during follow-up sessions with the patients.

To conclude, based on the findings of the present study, we recommend that chemoprophylaxis be considered for high-risk patients aged $\geq 40$ years who are undergoing knee arthroscopic surgery. This cut-off age was found to have a high NPV for the incidence of DVT in our local population. For patients undergoing arthroplasty, we recommend that the use of chemoprophylaxis be considered for high-risk patients, in accordance with the current AAOS guidelines.

\section{ACKNOWLEDGEMENT}

We would like to express our thanks to Ms Carmen Kam Jia Wen, Clinical Trials and Research Unit, Changi General Hospital, Singapore, for her help in and advice on the statistical analysis of this study.

\section{REFERENCES}

1. Falck-Ytter $\mathrm{Y}$, Francis CW, Johanson NA, et al; American College of Chest Physicians. Prevention of VTE in orthopedic surgery patients: Antithrombotic Therapy and Prevention of Thrombosis, 9th ed: American College of Chest Physicians Evidence-Based Clinical Practice Guidelines. Chest 2012; 141(2 Suppl):e278S-e325S.

2. Wirth T, Schneider B, Misselwitz F, et al. Prevention of venous thromboembolism after knee arthroscopy with low-molecular weight heparin (reviparin): Results of a randomized controlled trial. Arthroscopy 2001; 17:393-9

3. Michot M, Conen D, Holtz D, et al. Prevention of deep-vein thrombosis in ambulatory arthroscopic knee surgery: A randomized trial of prophylaxis with low--molecular weight heparin. Arthroscopy 2002; 18:257-63.

4. Demers C, Marcoux S, Ginsberg JS, et al. Incidence of venographically proved deep vein thrombosis after knee arthroscopy. Arch Intern Med 1998; 158:47-50.

5. Dong JT, Wang X, Men XQ, et al. Incidence of deep venous thrombosis in Chinese patients undergoing arthroscopic knee surgery for cruciate ligament reconstruction. Knee Surg Sports Traumatol Arthrosc 2014 Nov 2. [Epub ahead of print].

6. Sun Y, Chen D, Xu Z, et al. Deep venous thrombosis after knee arthroscopy: a systematic review and meta-analysis. Arthroscopy 2014; 30:406-12.

7. Ilahi OA, Reddy J, Ahmad I. Deep venous thrombosis after knee arthroscopy: a meta-analysis. Arthroscopy 2005; 21:727-30.

8. Williams JS Jr, Hulstyn MJ, Fadale PD, et al. Incidence of deep vein thrombosis after arthroscopic knee surgery: a prospective study. Arthroscopy $1995 ; 11: 701-5$

9. Dahl OE, Gudmundsen TE, Haukeland L. Late occurring clinical deep vein thrombosis in joint-operated patients. Acta Orthop Scand 2000; 71:47-50.

10. Graham WC, Flanigan DC. Venous thromboembolism following arthroscopic knee surgery: a current concepts review of incidence, prophylaxis, and preoperative risk assessment. Sports Med 2014; 44:331-43.

11. Ramos J, Perrotta C, Badariotti G, Berenstein G. Interventions for preventing venous thromboembolism in adults undergoing knee arthroscopy. Cochrane Database Syst Rev 2008; 8:CD005259.

12. White RH, Romano PS, Zhou H, Rodrigo J, Bargar W. Incidence and time course of thromboembolic outcomes following total hip or knee arthroplasty. Arch Intern Med 1998; 158:1525-31.

13. Lapidus LJ, Ponzer S, Pettersson H, de Bri E. Symptomatic venous thromboembolism and mortality in orthopaedic surgery - an observational study of 45968 consecutive procedures. BMC Musculoskelet Disord 2013; $14: 177$.

14. Bin Abd Razak HR, Tan HC. The use of pneumatic tourniquets is safe in Asians undergoing total knee arthroplasty without anticoagulation. Knee $2014 ; 21: 176-9$

15. Low MH, Yeo SJ, Chin PL, et al. A Singapore perspective on the use of a short course of chemothromboprophylaxis in patients who underwent total knee arthroplasty. Singapore Med J 2013; 54:560-3.

16. Lee WS, Kim KI, Lee HJ, Kyung HS, Seo SS. The incidence of pulmonary embolism and deep vein thrombosis after knee arthroplasty in Asians remains low: a meta-analysis. Clin Orthop Relat Res 2013; 471:1523-32. 